\title{
The supersymmetric extension of the Faddeev model
}

\author{
Lisa Freyhult* \\ Department of Theoretical Physics, Uppsala University \\ P.O. Box 803, S-75108, Uppsala, Sweden
}

\begin{abstract}
We study the supersymmetric extension of the Faddeev model in four dimensions. The Faddeev model contains three dimensional soliton solutions and we are interested in how these solitons are affected by supersymmetry. We consider both the $N=1$ and $N=2$ extensions and find that in neither case it is possible to supersymmetrize the model without adding additional bosonic terms. There are essentially two ways of constructing the supersymmetric theory, one that will lead to a model which allows for solitons and another that gives a model where solitons are excluded.

The $N=2$ model is studied since extending supersymmetry is the natural way of including topological charges in the algebra. A lower bound to the mass is obtained by computing the central charge. The result is that it is possible to have a non-trivial lower bound on the mass, this in principle allows for massive solitons.
\end{abstract}

\section{Introduction}

The Faddeev model [1] is a non-linear sigma model with higher derivative terms. It has been proposed as a low energy limit of Yang-Mills theory. The higher derivative terms makes three dimensional topological soliton solutions possible 2. Properties as a topological charge and a lower bound to the mass is associated to the solitons.

We will study the supersymmetric extension of the model. Supersymmetry might alter the properties of the solitons or even exclude them as solutions. We will consider if it is still possible to have the three dimensional solitons found in [2] in a supersymmetric model and, in that case, we will study their modified properties. One might also be interested in finding the corresponding fermionic solutions to the equations of motion.

We start by studying the $N=1$ supersymmetric extension to the model. This has essentially been discussed before in the context of supersymmetric skyrmeons [3. We review the results that we need here and explain how to translate the results in the context of the Faddeev model. We find that it is not possible to supersymmetrize the model as it stands, that is without adding any

*lisa.freyhult@teorfys.uu.se 
new bosonic terms to it. Considering solitons this could mean that the stability of the solutions is affected. We will essentially discuss two ways of writing the supersymmetric model. The action will contain fields that belong to the vector and hypermultiplet. Writing the action we will have the freedom of choosing the relative sign between the actions of the two multiplets. One of the choices will lead to a model where solitons are allowed, the other results in a model were they are excluded.

We then consider the $N=2$ supersymmetric model. This is motivated by that the central charge should give us a bound to the mass of our solutions. We also expect the eventual solutions to have nontrivial topological charge and extending supersymmetry is the natural way to include that charge in the algebra. New terms still has to be added to the original model in order to have supersymmetry, we discuss the options in the $N=2$ case. We compute the central charge and find that it will depend both on the fermionic and bosonic

fields. We consider the bosonic part of the central charge, which is relevant for bosonic solitons, and find a lower bound for the mass. The lower bound will depend on a topological invariant very similar to the Hopf invariant and from that we get a hint of what is required of a solution with nontrivial mass.

\section{The supersymmetric Faddeev model}

We choose to study the Faddeev model in 3+1 dimensions, this corresponds to the low energy limit of SU(2) Yang-Mills theory 11. The Lagrangian for the Faddeev model is [1] :

$$
L=\Lambda^{2} \partial_{\mu} n^{a} \partial^{\mu} n^{a}-\left(n^{a} \epsilon^{a b c} \partial_{\mu} n^{b} \partial_{\nu} n^{c}\right)^{2}
$$

where $n$ is a three component unit vector. We use the metric with signature (1 $-1-1-1)$. The second term is a closed two form squared and can be interpreted as $F_{\mu \nu}^{2}=\left(\partial_{\mu} A_{\nu}-\partial_{\nu} A_{\mu}\right)^{2}$ with:

$$
F_{\mu \nu}=n^{a} \epsilon^{a b c} \partial_{\mu} n^{b} \partial_{\nu} n^{c}
$$

Here $A_{\mu}$ can not locally be expressed in terms of $n$. The new variable $Z$ is therefore introduced. The vector $n$ is related to $Z$ as

$$
n^{a}=\bar{Z} T^{a} Z
$$

where $Z=\left(Z_{1}, Z_{2}\right)$ and $Z_{1,2}$ are complex scalar fields. We use $T^{a}$ to denote the generators of $\mathrm{SU}(2)$ in the fundamental representation. In terms of $Z$ the vector field and the field strength are:

$$
\begin{aligned}
& A_{\mu}=\frac{i}{2}\left(\bar{Z} \partial_{\mu} Z-\partial_{\mu} \bar{Z} Z\right) \\
& F_{\mu \nu}=i\left(\partial_{\mu} \bar{Z} \partial_{\nu} Z-\partial_{\nu} \bar{Z} \partial_{\mu} Z\right)
\end{aligned}
$$

The condition that $n$ is a unit vector is translated into

$$
\bar{Z} Z=1
$$


In terms of $Z$ the Lagrangian is written as:

$$
L=\Lambda^{2} \bar{D}_{\mu} \bar{Z} D^{\mu} Z-\left(\partial_{\mu} \bar{Z} \partial_{\nu} Z-\partial_{\nu} \bar{Z} \partial_{\mu} Z\right)^{2}
$$

with $D_{\mu}=\partial_{\mu}+i A_{\mu}$. Note that when introducing the variable $Z$ instead of $n$ we have introduced an extra degree of freedom, we could eliminate that degree of freedom by fixing the gauge of $A_{\mu}$.

Using $Z$ instead of $n$ is also more appropriate as $Z$ can be considered as one of the fields in the chiral multiplet. The chiral multiplet contains a complex scalar. Under supersymmetry the fields in the covariantly chiral multiplet transform in the following way:

$$
\begin{aligned}
\delta Z^{I} & =\sqrt{2} \xi^{\alpha} \psi_{\alpha}^{I} \\
\delta \psi_{\alpha}^{I} & =i \sqrt{2} \sigma_{\alpha \dot{\alpha}}^{\mu} \bar{\xi}^{\dot{\alpha}} D_{\mu} Z^{I}+\sqrt{2} \xi_{\alpha} F^{I} \\
\delta F^{I} & =i \sqrt{2} \bar{\xi}_{\dot{\alpha}} \bar{\sigma}^{\mu \dot{\alpha} \alpha} D_{\mu} \psi_{\alpha}^{I}-2 i \bar{\xi}_{\dot{\alpha}} Z^{I} \bar{\lambda}^{\dot{\alpha}}
\end{aligned}
$$

We have used capital latin letters to denote the components of $Z$, i.e. $I=1,2$. The vector field $A_{\mu}$ depends on the scalar field $Z$ but to start with we consider them as independent variables. We then expect $A_{\mu}$ to transform as part of the vector multiplet, the variations of the fields under supersymmetry are

$$
\begin{aligned}
& \delta A_{\mu}=i\left(\bar{\xi}_{\dot{\alpha}} \bar{\sigma}_{\mu}^{\dot{\alpha} \alpha} \lambda_{\alpha}+\xi^{\alpha} \sigma_{\mu \alpha \dot{\alpha}} \bar{\lambda}^{\dot{\alpha}}\right) \\
& \delta \lambda_{\alpha}=\frac{1}{2} \xi^{\beta} \sigma_{\beta \alpha}^{\mu \nu} F_{\mu \nu}+i \xi_{\alpha} D \\
& \delta D=\bar{\xi}_{\dot{\alpha}} \bar{\sigma}^{\mu \dot{\alpha} \alpha} \partial_{\mu} \lambda_{\alpha}-\xi^{\alpha} \sigma_{\alpha \dot{\alpha}}^{\mu} \partial_{\mu} \bar{\lambda}^{\dot{\alpha}}
\end{aligned}
$$

In order to find the supersymmetric theory we start by generalising the condition (6) by varying it with respect to supersymmetry. The following constraints are obtained.

$$
\begin{aligned}
& Z^{I} \bar{Z}_{I}=1 \\
& Z^{I} \bar{\psi}_{I}=0 \\
& Z^{I} \bar{F}_{I}=0 \\
& A_{\mu}=\frac{i}{2}\left(\partial_{\mu} \bar{Z}^{I} Z_{I}-\bar{Z}^{I} \partial_{\mu} Z_{I}\right)-\frac{1}{2} \psi^{I \alpha} \sigma_{\mu \alpha \dot{\alpha}} \bar{\psi}_{I}^{\dot{\alpha}} \\
& \lambda_{\alpha}=-\frac{1}{\sqrt{2}} i \bar{F}^{I} \psi_{I \alpha}+\frac{1}{\sqrt{2}} \sigma_{\alpha \dot{\alpha}}^{\mu} D_{\mu} Z^{I} \bar{\psi}_{I}^{\dot{\alpha}} \\
& D=\bar{D}^{\mu} \bar{Z}^{I} D_{\mu} Z_{I}+\frac{i}{2}\left(\psi^{I \alpha} \sigma_{\alpha \dot{\alpha}}^{\mu} \bar{D}_{\mu} \bar{\psi}_{I}^{\dot{\alpha}}-D^{\mu} \psi^{I \alpha} \sigma_{\mu \alpha \dot{\alpha}} \bar{\psi}_{I}^{\dot{\alpha}}\right)-\bar{F}^{I} F_{I}
\end{aligned}
$$

We see that the expression for $A_{\mu}$ in terms of $Z$, given in (4), follows from varying the constraint on $Z$. We could of course also have done it the other way around, imposing (4) and deriving the other expressions using the supersymmetric transformations. A fermionic term then has to be added to (4) for the vector field to satisfy the appropriate algebra. 
How to supersymmetrize the action above is in principle well known. The first part is similar to the nonlinear sigma model with gauge interactions and for the second part we write down the invariant action for the vector multiplet. Since these two parts of the action are supersymmetric independently of each other we are free to choose their relative sign. Hence we have

$$
\begin{gathered}
S=\Lambda^{2} \int d^{4} x\left(\bar{D}^{\mu} \bar{Z} D_{\mu} Z+i \psi \sigma^{\mu} \bar{D}_{\mu} \bar{\psi}-i D^{\mu} \psi \sigma_{\mu} \bar{\psi}-\bar{F} F+i \sqrt{2} \bar{Z} \lambda^{\alpha} \psi_{\alpha}\right. \\
\left.-i \sqrt{2} Z \bar{\lambda}_{\dot{\alpha}} \bar{\psi}^{\dot{\alpha}}+D(\bar{Z} Z-1)\right) \mp \int d^{4} x\left(F_{\mu \nu}^{2}+2 i \lambda \sigma^{\mu} \partial_{\mu} \bar{\lambda}-2 i \partial^{\mu} \lambda \sigma_{\mu} \bar{\lambda}-2 D^{2}\right)(20)
\end{gathered}
$$

If we now substitute for the fields in (17)-(19) we obtain the action in terms of the relevant fields. Note that when we do this we get, from the term that contains derivatives of the fermions in the vector multiplet, terms that contain derivatives of the field $F$. This means that the, previously, auxiliary fields become dynamical. Hence in order to make the model supersymmetric we have to add, additional to the fermions, another type of bosonic field. This is expected since in the original action (7) we have a term with more than two derivatives.

In the above we have introduced the freedom to choose the sign in front of the second part of the action. Comparing the supersymmetric action to the action of the bosonic model we choose the minus sign. This directly reproduces the terms in the bosonic action. The action in terms of the field $Z$ that is needed in order to construct the supersymmetric model is then

$$
S=\int d^{4} x\left(\Lambda^{2} \bar{D}^{\mu} \bar{Z} D_{\mu} Z-F_{\mu \nu}^{2}+2\left(\bar{D}^{\mu} \bar{Z} D_{\mu} Z\right)^{2}\right)
$$

The last term comes from the $D^{2}$ in $(20)$. The conclusion is that the Faddeev model can not be supersymmetrized as it stands, another term has to be added. This term is fourth order in time derivatives and the model therefore lacks an hamiltonian interpretation.

What is more is that when considering solitons in this model one will find that the new term acts unstabilizing. This is seen by considering the energy of a static field configuration and applying Derrick's theorem [7] to it. To make this a little more transparent we go back to the original variables, $n$. The action (21) is then

$$
S=\int d^{4} x\left(\Lambda^{2} \partial^{\mu} n \partial_{\mu} n-\left(\partial^{\mu} n \times \partial^{\nu} n\right)\left(\partial_{\mu} n \times \partial_{\nu} n\right)+2\left(\partial^{\mu} n \partial_{\mu} n\right)^{2}\right)
$$

Since we are interested in how the new term affects solitons in the model we consider the energy for static fields

$$
\begin{aligned}
E & =\int d^{3} x\left(\Lambda^{2} \partial_{i} n \partial_{i} n+F_{i j}^{2}-2\left(\partial_{i} n \partial_{i} n\right)^{2}\right) \\
& =\int d^{3} x\left(\Lambda^{2} \partial_{i} n \partial_{i} n+\left(\partial_{i} n \partial_{i} n\right)^{2}-\left(\partial_{i} n \partial_{j} n\right)^{2}-2\left(\partial_{i} n \partial_{i} n\right)^{2}\right) \\
& =\int d^{3} x\left(\Lambda^{2} \partial_{i} n \partial_{i} n-\left(\partial_{i} n \partial_{i} n\right)^{2}-\left(\partial_{i} n \partial_{j} n\right)^{2}\right)
\end{aligned}
$$


We now apply Derrick's theorem to (24). One finds that as $x \rightarrow \lambda x$ the term proportional to $\Lambda^{2}$, we call it $E_{2}$, scales as $\lambda$ and the other terms, we call them $E_{4}$ scales as $1 / \lambda$. If $\lambda=1$ corresponds to a minimum of the energy we have that

$$
0=\left.\frac{d E}{d \lambda}\right|_{\lambda=1}=\left.\frac{d}{d \lambda}\left(\lambda E_{2}-\frac{1}{\lambda} E_{4}\right)\right|_{\lambda=1}=E_{2}+E_{4}
$$

Both $E_{2}$ and $E_{4}$ are positive definite and hence they both have to be zero for the above to hold. Hence soliton solutions are not possible in the model.

There is a possibility to restore the loss of stability in the model by adding other terms that are also fourth order in derivatives and at the same time keeping supersymmetry, see 3 . In terms of $Z$ the terms that we could add would be $-(\bar{D} \bar{Z} D Z)^{2}+\square \bar{Z} \square Z$ times some arbitrary constant. We will not pursue this direction and refer to [3] for further details. We will instead go back to the action (20) and choose the other possibility for the sign in front of the second part of the action. The action in terms of the original variable, $Z$, is then

$$
S=\int d^{4} x\left(\Lambda^{2} \bar{D}^{\mu} \bar{Z} D_{\mu} Z+F_{\mu \nu}^{2}-2\left(\bar{D}^{\mu} \bar{Z} D_{\mu} Z\right)^{2}\right)
$$

The corresponding energy in terms of $n$ is

$$
\begin{aligned}
& E=\int d^{3} x\left(\Lambda^{2} \partial_{i} n \partial_{i} n+\left(\partial_{i} n \partial_{i} n\right)^{2}+\left(\partial_{i} n \partial_{j} n\right)^{2}\right) \\
& =\int d^{3} x\left(\Lambda^{2} \partial_{i} n \partial_{i} n+F_{i j}^{2}+2\left(\partial_{i} n \partial_{j} n\right)^{2}\right)
\end{aligned}
$$

Hence we see that this is an alternative way to write the extension of the Faddeev model. Applying Derrick's theorem to this expression solitons are not ruled out.

Summarizing we can not obtain a supersymmetric version of the Faddeev model. We have to add terms that are fourth order in time derivatives. Both (21) and (26) are models that can be supersymmetrized and they both contain the action of the Faddeev model plus a term that is fourth order in derivatives. In (26) solitons are possible while in (21) they are not.

The energy of the full model corresponding to (26) is

$$
\begin{array}{r}
E=\Lambda^{2} \int d^{4} x\left(\bar{D}_{i} \bar{Z} D_{i} Z+i \psi \sigma_{i} \bar{D}_{i} \bar{\psi}-i D_{i} \psi \sigma_{i} \bar{\psi}+\bar{F} F-i \sqrt{2} \bar{Z} \lambda^{\alpha} \psi_{\alpha}\right. \\
\left.+i \sqrt{2} Z \bar{\lambda}_{\dot{\alpha}} \bar{\psi}^{\dot{\alpha}}-D(\bar{Z} Z-1)\right)+\int d^{4} x\left(-F_{i j}^{2}+2 i \lambda \sigma_{i} \partial_{i} \bar{\lambda}-2 i \partial_{i} \lambda \sigma_{i} \bar{\lambda}+2 D^{2}\right)
\end{array}
$$

With

$$
\begin{aligned}
& A_{i}=\frac{i}{2}\left(\partial_{i} \bar{Z} Z-\bar{Z} \partial_{i} Z\right)-\frac{1}{2} \psi^{\alpha} \sigma_{i \alpha \dot{\alpha}} \bar{\psi}^{\dot{\alpha}} \\
& \lambda_{\alpha}=-\frac{1}{\sqrt{2}} i \bar{F} \psi_{\alpha}-\frac{1}{\sqrt{2}} \sigma_{i \alpha \dot{\alpha}} D_{i} Z \bar{\psi}^{\dot{\alpha}} \\
& D=-\bar{D}_{i} \bar{Z} D_{i} Z-\frac{i}{2}\left(\psi^{\alpha} \sigma_{i \alpha \dot{\alpha}} \bar{D}_{i} \bar{\psi}^{\dot{\alpha}}+D_{i} \psi^{\alpha} \sigma_{i \alpha \dot{\alpha}} \bar{\psi}^{\dot{\alpha}}\right)-\bar{F} F
\end{aligned}
$$


We obtain a lower bound to the energy of the model as

$$
\begin{aligned}
& E \geq \int d^{3} x\left(\Lambda^{2} \partial_{i} n \partial_{i} n+F_{i j}^{2}+2\left(\partial_{i} n \partial_{j} n\right)^{2}\right) \\
& \geq \int d^{3} x\left(\Lambda^{2} \partial_{i} n \partial_{i} n+F_{i j}^{2}\right) \geq K Q_{H}^{3 / 4}
\end{aligned}
$$

The last inequality we get following $[8$ and 9$]$. Here $Q_{H}$ is the Hopf invariant and $K$ is a constant. The Hopf invariant is given by

$$
Q_{H}=\frac{1}{(8 \pi)^{2}} \int d^{3} x \epsilon^{i j k} F_{i j} A_{k}
$$

Hence if the solitons have a nontrivial Hopf invariant their mass is nonzero. Note that this is the same bound to the mass as in the non-supersymmetric model 8 9. It is possible that the other terms affects the bound so that it could be higher in the supersymmetric model. However it is not easy to say because of the form of the terms. Here we are just interested in finding some bound which can give a nontrivial mass, for that the above is enough.

\section{$3 \quad \mathrm{~N}=2$ supersymmetric model}

The proper way of introducing topological charges in supersymmetric theories is to consider extended supersymmetry [5]. We saw that the bosonic part of the $N=1$ model, as well as the original bosonic model, gave a bound to the mass that was proportional to the Hopf invariant to the power $3 / 4$. In extended supersymmetry a lower bound to the mass is proportional to the central charge in the model [5]. Hence we expect a mass bound that is linear in the topological invariant for the $N=2$ model, something that seems to be very different from what we had in the $N=1$ model. We do not expect a linear bound from the Faddeev model and it is therefore interesting to study this case where it seems that we get just that. We therefore proceed and construct the $N=2$ version of the Faddeev model.

The variations of the fields in the $N=2$ hypermultiplet coupled to the vector multiplet are

$$
\begin{aligned}
& \delta Z^{I}=\sqrt{2} \xi^{\alpha I} \psi_{\alpha} \\
& \delta \psi_{\alpha}=i \sqrt{2} \sigma_{\alpha \dot{\alpha}}^{\mu} \bar{\xi}^{\dot{\alpha} I} D_{\mu} Z_{I}+i \sqrt{2} \phi \xi_{\alpha}^{I} \bar{Z}_{I}+\sqrt{2} \xi_{\alpha}^{I} F_{I} \\
& \delta F^{I}=i \sqrt{2} \bar{\xi}_{\dot{\alpha}}^{I} \bar{\sigma}^{\mu \dot{\alpha} \alpha} D_{\mu} \psi_{\alpha}-i \sqrt{2} \bar{\xi}_{\dot{\alpha}}^{I} \phi \bar{\psi}^{\dot{\alpha}}-2 i \bar{\xi}_{\dot{\alpha}}^{I} \bar{\lambda}^{\alpha K^{K}} Z_{K} \\
& \delta A_{\mu}=i\left(\bar{\xi}_{\dot{\alpha}}^{I} \bar{\sigma}_{\mu}^{\dot{\alpha} \alpha} \lambda_{I \alpha}+\xi^{\alpha I} \sigma_{\mu \alpha \dot{\alpha}} \bar{\lambda}_{I}^{\dot{\alpha}}\right) \\
& \delta \phi=2 \xi^{\alpha I} \lambda_{I \alpha} \\
& \delta \lambda_{\alpha}^{I}=\frac{1}{2} \xi^{\beta I} \sigma_{\beta \alpha}^{\mu \nu} F_{\mu \nu}+i \sigma_{\alpha \dot{\alpha}}^{\mu} \partial_{\mu} \phi \bar{\xi}^{\dot{\alpha} I}+i \xi_{\alpha}^{I} D \\
& \delta D=\bar{\xi}_{\dot{\alpha}}^{I} \bar{\sigma}^{\mu \dot{\alpha} \alpha} \partial_{\mu} \lambda_{I \alpha}-\xi^{\alpha I} \sigma_{\alpha \dot{\alpha}}^{\mu} \partial_{\mu} \bar{\lambda}_{I}^{\dot{\alpha}}
\end{aligned}
$$


Varying the constraint (6) under this new supersymmetry we obtain expressions for the fields in the vector multiplet in terms of the fields in the hypermultiplet

$$
\begin{aligned}
& \bar{Z}^{I} Z_{I}=1 \\
& \bar{\xi}_{\dot{\alpha}}^{I} \bar{\psi}^{\dot{\alpha}} Z_{I}+\xi^{I \alpha} \psi_{\alpha} \bar{Z}_{I}=0 \\
& A_{\mu}=\frac{i}{2}\left(\partial_{\mu} \bar{Z}^{I} Z_{I}-\bar{Z}^{I} \partial_{\mu} Z_{I}\right)-\frac{1}{2} \psi^{\alpha} \sigma_{\mu \alpha \dot{\alpha}} \bar{\psi}^{\dot{\alpha}} \\
& \phi=-i \bar{F}^{I} Z_{I} \\
& \lambda_{\alpha}^{I}=-\frac{i}{\sqrt{2}} \psi_{\alpha} \bar{F}^{I}-\frac{1}{\sqrt{2}} Z^{I} \sigma_{\alpha \dot{\alpha}}^{\mu} \bar{D}_{\mu} \bar{\psi}^{\dot{\alpha}}-Z^{I} \phi^{\dagger} \psi_{\alpha} \\
& D=\bar{D}^{\mu} \bar{Z}^{I} D_{\mu} Z_{I}+\frac{i}{2}\left(\psi^{\alpha} \sigma_{\alpha \dot{\alpha}}^{\mu} \bar{D}_{\mu} \bar{\psi}^{\dot{\alpha}}-D^{\mu} \psi^{\alpha} \sigma_{\mu \alpha \dot{\alpha}} \bar{\psi}^{\dot{\alpha}}\right)-\bar{F}^{I} F_{I} \\
& -\frac{i}{2} \psi^{\alpha} \phi^{\dagger} \psi_{\alpha}+\frac{i}{2} \bar{\psi}_{\dot{\alpha}} \phi \bar{\psi}^{\dot{\alpha}}+\bar{Z}^{I} \phi^{\dagger} \phi Z_{I}+\sqrt{2} i \bar{Z}^{I} \lambda_{I} \psi-\sqrt{2} i Z^{I} \bar{\lambda}_{I} \bar{\psi}
\end{aligned}
$$

The action of the $N=2$ model with a hypermultiplet in interaction with the vector multiplet is

$$
\begin{aligned}
& S=\Lambda^{2} \int d^{4} x\left(\bar{D}^{\mu} \bar{Z} D_{\mu} Z+\frac{i}{2} \psi \sigma^{\mu} \bar{D}_{\mu} \bar{\psi}-\frac{i}{2} D^{\mu} \psi \sigma_{\mu} \bar{\psi}-\bar{F} F+\sqrt{2} i \bar{Z} \lambda \psi\right. \\
& \left.-\sqrt{2} i Z \bar{\lambda} \bar{\psi}-\frac{i}{2} \psi^{\alpha} \phi^{\dagger} \psi_{\alpha}+\frac{i}{2} \bar{\psi}_{\dot{\alpha}} \phi \bar{\psi}^{\dot{\alpha}}+\bar{Z} \phi^{\dagger} \phi Z+D(\bar{Z} Z-1)\right) \\
& \pm 4 \int d^{4} x\left(\partial^{\mu} \phi^{\dagger} \partial_{\mu} \phi-\frac{1}{4} F_{\mu \nu} F^{\mu \nu}+\frac{i}{2} \partial^{\mu} \lambda \sigma_{\mu} \bar{\lambda}-\frac{i}{2} \lambda \sigma^{\mu} \partial_{\mu} \bar{\lambda}+\frac{D^{2}}{2}\right)
\end{aligned}
$$

Note that the action is supersymmetric independently of the sign in front of the terms in the action that contains the vectormultiplet. We saw in the $N=1$ case that the stability of solitons in the model was dependent on exactly that sign.

Using the expressions (43)-(48) the Lagrangian can be written in terms of the fields in the hypermultiplet. The action that is needed to construct the $N=2$ model is

$$
S=\int d^{4} x\left(\Lambda^{2} \bar{D}^{\mu} \bar{Z} D_{\mu} Z \mp F^{\mu \nu} F_{\mu \nu} \pm 2\left(\bar{D}^{\mu} \bar{Z} D_{\mu} Z\right)^{2}\right)
$$

The last term comes from the $D^{2}$-term in (49). This is the same action that was needed in order to construct the $N=1$ model. It seems that, analogous to the $N=1$ model, by choosing the appropriate sign we can have stable solitons in the model. Note however that there are other terms in the Lagrangian now that can make the model unstable. We want to study how the solitons in the original model are affected and we therefore proceed and consider bosonic solitons in the supersymmetric model. We restrict our considerations to bosonic solitons for simplicity. The previously auxiliary field, $F$, is now a dynamical field which means that we have more than one type of bosons to take into account. Some of the terms that contain $F$ are negative energy terms which is the reason for our concern. 
The full bosonic action is

$$
\begin{aligned}
& S_{\text {bosonic }}=\Lambda^{2} \int d^{4} x\left(\bar{D}^{\mu} \bar{Z} D_{\mu} Z-\bar{F} F+|\bar{Z} F|^{2}\right) \pm 4 \int d^{4} x\left(-\frac{1}{4} F^{\mu \nu} F_{\mu \nu}\right. \\
& \left.+\partial^{\mu}(\bar{Z} F) \partial_{\mu}(Z \bar{F})+\frac{1}{2}\left(\bar{D}^{\mu} \bar{Z} D_{\mu} Z-\bar{F} F+|\bar{Z} F|^{2}\right)^{2}\right)
\end{aligned}
$$

The corresponding energy is

$$
\begin{aligned}
& E_{\text {bosonic }}=\Lambda^{2} \int d^{3} x\left(\bar{D}_{i} \bar{Z} D_{i} Z+\bar{F} F-|\bar{Z} F|^{2}\right)+4 \int d^{3} x\left( \pm \frac{1}{4} F_{i j} F_{i j}\right. \\
& \left. \pm \partial_{i}(\bar{Z} F) \partial_{i}(Z \bar{F}) \mp \frac{1}{2}\left(-\bar{D}_{i} \bar{Z} D_{i} Z-\bar{F} F+|\bar{Z} F|^{2}\right)^{2}\right)
\end{aligned}
$$

We will choose the sign such that the original bosonic energy, that we need in order to be able to have supersymmetry, allows for soliton solutions. We do this because we do not want to exclude the possibility of having solitons in terms of the field $Z$ only. Considering the full bosonic theory we are interested in how the interactions with the new bosonic field affects the stability and mass bound of the solitons. It is difficult to find a mass bound directly from the energy (52) since one cannot directly determine the relative size of the terms containing $F$. However in an extended supersymmetric theory we know that we can compute the central charge and from that obtain a mass bound [5]. This means that there is an indirect way to find the mass bound, we do not have to make an estimate from (52).

\section{A mass bound}

We now proceed with an attempt to compute the central charge of the $N=2$ algebra above. The computation is well known, see for example [6], in the case where the fields in the vector multiplet are independent of those in the scalar multiplet. Here however we also have to take (43)-(48) into account. Note that in the following we have made the choice of a negative sign in front of the second part of the action, corresponding to the action of the vector multiplet.

The generators of supersymmetry are

$$
\begin{aligned}
& Q_{1}=\int d^{3} x(-4)\left(\sigma^{\mu} \bar{\lambda}_{1}\left(i F_{0 \mu}+\tilde{F}_{0 \mu}\right)+\sigma^{\mu} \bar{\sigma}^{0} \lambda_{2} \partial_{\mu} \phi^{\dagger}+\sigma^{0} \bar{\lambda}_{1} D\right) \\
& +\sqrt{2} \Lambda^{2}\left(\sigma^{\mu} \bar{\sigma}^{0} \psi \bar{D}_{\mu} \bar{Z}_{1}+\sigma^{0} \bar{\psi} F_{2}+\sigma^{0} Z_{1} \phi^{\dagger} \bar{\psi}+\frac{1}{\sqrt{2}} \bar{\sigma}^{0} \bar{\lambda}_{1} \bar{Z} Z\right) \\
& Q_{2}=\int d^{3} x(-4)\left(\sigma^{\mu} \bar{\lambda}_{2}\left(i F_{0 \mu}+\tilde{F}_{0 \mu}\right)-\sigma^{\mu} \bar{\sigma}^{0} \lambda_{1} \partial_{\mu} \phi^{\dagger}+\sigma^{0} \bar{\lambda}_{2} D\right) \\
& +\sqrt{2} \Lambda^{2}\left(\sigma^{\mu} \bar{\sigma}^{0} \psi \bar{D}_{\mu} \bar{Z}_{2}-\sigma^{0} \bar{\psi} F_{1}+\sigma^{0} Z_{2} \phi^{\dagger} \bar{\psi}+\frac{1}{\sqrt{2}} \bar{\sigma}^{0} \bar{\lambda}_{2} \bar{Z} Z\right)
\end{aligned}
$$

together with their complex conjugates. The dual field strength tensor is given by $\tilde{F}^{\mu \nu}=\frac{1}{2} \epsilon^{\mu \nu \rho \sigma} F_{\rho \sigma}$. The algebra of the $N=2$ model is

$$
\left\{\tilde{Q}_{\alpha}^{I}, \overline{\tilde{Q}}_{\dot{\beta}}^{J}\right\}=2 i \sigma_{\alpha \dot{\beta}}^{\mu} P_{\mu} \delta^{I J}
$$




$$
\left\{\tilde{Q}_{\alpha}^{I}, \tilde{Q}_{\beta}^{J}\right\}=2 Z^{I J} \epsilon_{\alpha \beta}
$$

where $\tilde{Q}_{1}=\frac{1}{\sqrt{2}}\left(Q_{1}+i Q_{2}\right)$ and $\tilde{Q}_{2}=\frac{1}{\sqrt{2}}\left(Q_{1}-i Q_{2}\right)$. Here $Z^{I J}$ is antisymmetric in its indices and called the central charge. The central charge gives a mass bound to the eventual solitons in the model $[5]$ according to

$$
M_{S U S Y} \geq \sqrt{2}\left|Z_{12}\right|
$$

We therefore want to compute the anticommutator in (56). We do that using

$$
\begin{aligned}
& \left\{\lambda_{\alpha}^{I}, \bar{\lambda}_{J \dot{\beta}}\right\}=\frac{i}{4} \sigma_{\alpha \dot{\beta}}^{0} \delta_{J}^{I} \\
& \left\{\psi_{\alpha}, \bar{\psi}_{\dot{\beta}}\right\}=\frac{i}{\Lambda^{2}} \sigma_{\alpha \dot{\beta}}^{0}
\end{aligned}
$$

and the corresponding commutators for the scalar and vector field. Assuming that we have static bosonic solitons in the model we obtain for the central charge:

$$
\left\{Q_{\alpha}^{1}, Q_{\beta}^{2}\right\}=8 i \int d^{3} x \frac{1}{2} \epsilon^{i j k} F_{j k} \partial_{i} \phi^{\dagger} \epsilon_{\alpha \beta}
$$

Hence we have that the central charge is

$$
Z^{12}=2 i \int d^{3} x \epsilon^{i j k} F_{j k} \partial_{i} \phi^{\dagger}
$$

Where $F_{i j}$ is the bosonic part of the field strength, $F_{i j}=i\left(\partial_{i} \bar{Z} \partial_{j} Z-\partial_{j} \bar{Z} \partial_{i} Z\right)$, and $\phi$ is given by (46). Supersymmetry gives us the following relation for the mass of solitons in the model

$$
M_{S U S Y} \geq \sqrt{2}\left|Z^{12}\right|=\sqrt{2}\left|2 i \int d^{3} x \epsilon^{i j k} F_{j k} \partial_{i} \phi^{\dagger}\right|=2 \sqrt{2}\left|\int d^{3} x \epsilon^{i j k} F_{j k} \partial_{i}\left(\bar{Z}^{I} F_{I}\right)\right|
$$

In order to see if the lowest bound is non-zero we study the vacuum configuration. We start by considering the terms in the action that corresponds to a potential

$$
V(Z, F)=\Lambda^{2}(F \bar{F}-(\bar{Z} F)(\bar{F} Z))+2(F \bar{F}-(\bar{Z} F)(\bar{F} Z))^{2}
$$

Since $F \bar{F}-(\bar{Z} F)(\bar{F} Z) \geq 0$, the potential is positive definite and we have that the energy is minimized when $V(Z, F)=0$. This corresponds to a field configuration where

$$
F \bar{F}-(\bar{Z} F)(\bar{F} Z)=0 \quad \Leftrightarrow \quad Z \| F, \quad|F|=|\bar{Z} F|
$$

That is we have

$$
\bar{Z} F=C e^{i \chi} \quad F_{I}=C Z_{I} e^{i \chi}
$$

Where $C=$ constant is a special solution. In order to compute the mass bound we introduce the parametrization of $Z$ :

$$
Z=\left(\begin{array}{l}
e^{i \Phi_{12}} \sin \frac{\nu}{2} \\
e^{i \Phi_{34}} \cos \frac{\nu}{2}
\end{array}\right)
$$


We define $\alpha=\Phi_{12}+\Phi_{34}, \beta=\Phi_{34}-\Phi_{12}$ and $\gamma=\pi-\nu$ and obtain for the bosonic part of the vector field and field strength

$$
A=\cos \gamma d \beta+d \alpha \quad F=\sin \gamma d \beta \wedge d \gamma
$$

Using this we obtain for the mass bound

$$
M_{S U S Y} \geq\left|\sqrt{2} C \int \sin \gamma d \beta \wedge d \gamma \wedge d \chi\right|
$$

If $\gamma, \beta$ and $\chi$ are coordinates on $S^{3}$ this is, up to a constant, the winding number $\pi_{3}\left(S^{3}\right)$. Hence if the angle $\chi$ is nontrivial we have a nontrivial bound to the mass of solutions to the model. Note the similarity of the mass bound to the Hopf invariant

$$
Q_{H} \propto \int F \wedge A=\int \sin (\gamma) d \beta \wedge d \gamma \wedge d \alpha
$$

The Hopf invariant is not directly related to the central charge in this model we just note that the structure of the two invariants are very similar. However the above suggest that there are possibilities to have solitons with nontrival mass. In order to find out exactly which masses we can have in the model we have to explicitly find the solitons.

The above suggests that the bound is linear in the topological invariant, just as expected. However the topological invariant that we obtained in the supersymmetric model is not necessarily the same as the Hopf invariant in the bosonic model. In order to better understand how the two invariants are related we will compare the two expressions when the bounds are saturated. We begin by reviewing how the bound is obtained in the ordinary Faddeev model. The energy of the Faddeev model is

$$
E=e_{2}+e_{4}
$$

where $e_{2}=\Lambda^{2}\left(\partial_{i} n\right)^{2}$ and $e_{4}=F_{i j}^{2}=\left(n \cdot \partial_{i} n \times \partial_{j} n\right)^{2}$. The energy can be given a lower bound as follows

$$
E=\left(\sqrt{e_{2}}-\sqrt{e_{4}}\right)^{2}+2 \sqrt{e_{2}} \sqrt{e_{4}} \geq 2 \sqrt{e_{2}} \sqrt{e_{4}}
$$

This is then related to by a Sobolev-type inequality to an expression proportional the Hopf invariant to the power $3 / 4[\underline{8}, 9$. The bound above is saturated when $\sqrt{e_{2}}=\sqrt{e_{4}}$. This is consistent with the result from Derrick's theorem. The energy is then

$$
E_{\min }=2 e_{4}
$$

We will now use the bound (62) that we obtained for the energy of the bosonic part of the supersymmetric model. Completing the square the energy (52) can be written as

$$
\begin{aligned}
& E_{\text {bosonic }}=\int d^{3} x\left(\Lambda^{2}\left(\bar{D}_{i} \bar{Z} D_{i} Z+\bar{F} F-|\bar{Z} F|^{2}\right)\right. \\
& -\left(\frac{1}{\sqrt{2}} \epsilon_{i j k} F_{j k}+2 i \partial_{i}(\bar{Z} F)\right)\left(\frac{1}{\sqrt{2}} \epsilon_{i j k} F_{j k}-2 i \partial_{i}(Z \bar{F})\right) \\
& \left.+\sqrt{2} \epsilon_{i j k} F_{j k}\left(i \partial_{i}(\bar{Z} F)-i \partial_{i}(Z \bar{F})\right)+2\left(\bar{D}_{i} \bar{Z} D_{i} Z+\bar{F} F-|\bar{Z} F|^{2}\right)^{2}\right)
\end{aligned}
$$


We have that the energy is

$$
E_{\text {bosonic }}=2 \sqrt{2}\left|\int d^{3} x \epsilon_{i j k} F_{j k} \partial_{i}(\bar{Z} F)\right|
$$

when the following equation holds

$$
\begin{array}{r}
\int d^{3} x\left(\Lambda^{2}\left(\bar{D}_{i} \bar{Z} D_{i} Z+\bar{F} F-|\bar{Z} F|^{2}\right)+2\left(\bar{D}_{i} \bar{Z} D_{i} Z+\bar{F} F-|\bar{Z} F|^{2}\right)^{2}\right. \\
\left.-\left(\frac{1}{\sqrt{2}} \epsilon_{i j k} F_{j k}+2 i \partial_{i}(\bar{Z} F)\right)\left(\frac{1}{\sqrt{2}} \epsilon_{i j k} F_{j k}-2 i \partial_{i}(Z \bar{F})\right)\right)=0
\end{array}
$$

One configuration that saturates this bound is

$$
\begin{aligned}
& \frac{1}{\sqrt{2}} \epsilon_{i j k} F_{j k}+2 i \partial_{i}(\bar{Z} F)=0 \\
& V(Z, F)=0 \\
& \bar{D}_{i} \bar{Z} D_{i} Z=0
\end{aligned}
$$

Using this the energy, when the bound is saturated, is

$$
E_{\text {min }}=\left|\int d^{3} x \epsilon_{i j k} F_{j k} \epsilon_{i l m} F_{l m}\right|=2 e_{4}
$$

This coincides with the minimal energy for the original bosonic Faddeev model. Hence the original bound is contained in the supersymmetric bound. Therefore, if the bound is nontrivial in the original model the bound must also be nontrivial in the supersymmetric model.

\section{Conclusions}

In conclusion we have found that it is not possible to supersymmetrize the Faddeev model as it stands. We have to add new higher derivative terms to make it possible. There are several ways to modify the model to get a version that can be supersymmetrized. We have analyzed the different possibilities and found that there is one version of the model that is supersymmetric but where bosonic solitons are excluded and one where they are allowed. We have considered both the $N=1$ and the $N=2$ versions of the model and found that the original theory have to be modified in the same way in both cases to allow for supersymmetry. A lower bound to the mass have been found in both models. In the $N=1$ case the bound is proportional to the Hopf invariant to the power $3 / 4$. In the $N=2$ case the bound is given by an invariant very similar, but not directly related, to the Hopf invariant. This bound is, as expected in a theory with extended supersymmetry, linear in the topological invariant. We have compared the saturated bounds in the different models and found that the bound in the extended model contains the non-supersymmetric and $\mathrm{N}=1$

bound. Doing that we draw the conclusion that a nontrivial lower bound is 
possible in the model with extended supersymmetry just as in $N=1$ and the non-supersymmetric model.

Here we have mainly considered bosonic solitons for the reason that in the ordinary Faddeev model three dimensional solitons exist. We have analyzed how these solitons are affected when supersymmetry is added. It would also be of interest to find the corresponding fermionic solitons in the model and study how the interaction between fermions and bosons affects the solutions. This would presumably be quite involved since one would have to solve the fermionic equations of motion in the background of the bosonic solitons. Due to the non-linearity of the equations the fermionic solutions would affect the bosonic etc. What is easily done however is to compute a bound on the mass for the full model, containing fermions. It might from there be possible to draw some conclusions of what is required of a fermionic soliton for it to contribute to the mass bound. This remains for future study.

\section{Acknowledgements}

I would like to thank A.J. Niemi for helpful discussions and for reading the manuscript. I would also like to thank S.F. Hassan, U. Lindström, N.S. Manton and P. Sundell for useful discussions.

\section{References}

[1] L. D. Faddeev and A. J. Niemi, Phys. Rev. Lett. 82 (1999) 1624 arXiv:hep-th/9807069 . L. D. Faddeev and A. J. Niemi, Phys. Lett. B 525 (2002) 195 arXiv:hep-th/0101078. L. D. Faddeev and A. J. Niemi, Phys. Lett. B 449 (1999) 214 arXiv:hep-th/9812090.

[2] L. D. Faddeev and A. J. Niemi, Nature $\mathbf{3 8 7} \quad$ (1997) 58 arXiv:hep-th/9610193.

[3] E. A. Bergshoeff, R. I. Nepomechie and H. J. Schnitzer, Nucl. Phys. B 249 (1985) 93.

[4] L. D. Faddeev, Print-75-0570 (IAS, PRINCETON)

[5] E. Witten and D. I. Olive, Phys. Lett. B 78 (1978) 97.

[6] L. Alvarez-Gaume and S. F. Hassan, Fortsch. Phys. 45 (1997) 159 arXiv:hep-th/9701069.

[7] G. H. Derrick, J. Math. Phys. 5 (1964) 1252.

[8] A. F. Vakulenko and L. V. Kapitansky, Sov. Phys. Dokl. 24 (1979) 433.

[9] R. S. Ward, Nonlinearity. 12 (1999) 241 arXiv:hep-th/9811176

[10] M. F. Sohnius, Phys. Rept. 128 (1985) 39. 